\title{
Metagenomic analysis of microbial community in over-fermented tempeh
}

\author{
ARTINI PANGASTUTI", RIZAL KHOIRUN ALFISAH, NUR IFFAH ISTIANA, SITI LUSI ARUM SARI, \\ RATNA SETYANINGSIH, ARI SUSILOWATI, TJAHJADI PURWOKO \\ Microbe Biomaterials Research Group, Department of Biology, Faculty of Mathematics and Natural Sciences, Universitas Sebelas Maret. Ir. Sutami 36A \\ Surakarta 57126, Central Java, Indonesia. Tel./fax. +62-271-663375, `email: artini_p@staff.uns.ac.id
}

Manuscript received: 27 October 2018. Revision accepted: 24 March 2019.

\begin{abstract}
Pangastuti A, Alfisah RK, Istiana NI, Sari SLA, Setyaningsih R, Susilowati A, Purwoko T. 2019. Metagenomic analysis of microbial community in over-fermented tempeh. Biodiversitas 20: 1106-1114. Tempeh is a traditional Indonesian food which is made from soybeans through a fermentation process using Rhizopus as a starter culture. Tempeh is now considered as a functional food with many beneficial effects to human health beyond its nutritional value. The microbial community during the further fermentation process of tempeh give typical characteristic taste and flavor. Therefore the over-fermented tempeh is used as a flavoring in some dishes. Metagenomic analysis needed to know the involvement of microbial communities since most of the microbes involved in further fermentation process are unculturable. This research aimed to study the diversity of the microbial community in the over-fermented tempeh (72 hours) using the metagenomic analysis. Seventeen OTUs of fungi in over-fermented tempeh were detected. Among them, 9 OTUs had significant abundance: six species were identified as Tryblidiopsis sichuanensis, Candida sp.2_1., Kluyveromyces marxianus, Trichosporon asahii, Trichosporon gracile, and Trichosporon ovoides, one species was identified in the order level Mucorales, and two fungi species could not be determined. Species of the order Mucorales was the dominant species in over-fermented tempeh (72 hours) with a relative abundance of $62.46 \%$, followed by Kluyveromyces marxianus with a relative abundance of $3698 \%$. Meanwhile, 132 OTUs of the bacterial community was detected, the predominant 10 genera were Chryseobacterium, Lactococcus, Lactobacillus, Streptococcus, Acetobacter, Novosphingobium, Comamonas, Escherichia, Klebsiella, and Stenotrophomonas, in which Lactobacillus agilis, Lactococcus sp., and Klebsiella sp., were most abundant with relative abundance of $27 \%, 26.3 \%$, and $13 \%$ respectively. Microorganisms in over fermented tempeh were thought to play a role in the degradation of organic compounds and the formation of aromas and flavors. This information will be useful for controlling the production of over fermented tempeh as an alternative flavoring agent.
\end{abstract}

Keywords: Fungi metagenomic, next-generation sequencing, over-fermented tempeh

\section{INTRODUCTION}

Tempeh is a traditional Indonesian food made from soybeans through a fermentation process, using the fungus Rhizopus oligosporus as a key fungus. The microbial process during tempeh fermentation makes this food have many beneficial health effects beyond its nutritional value. Utilization of tempeh in Indonesia is not only in the form of fresh tempeh but also over-fermented tempeh which used as a flavoring in traditional Indonesian cuisine. For this purpose, the tempeh is further fermented until 72 hours, and called 'tempe bosok' or 'rotten tempeh'. Approximately $20 \%$ of tempeh consumed in Java is overfermented tempeh (Shurtleff and Aoyagi 1979).

Over-fermented tempeh has a typical texture, taste, and flavor, which are formed depending on the fermentation process, soybeans used, the boiling process and the microbial consortium (Utami et al. 2016). During tempeh fermentation, complex organic matters were hydrolyzed by the microorganisms. The free amino acid content increased during the over-fermentation of tempeh (Handoyo and Morita 2006). Amino acids which known to contribute to developing umami taste, glutamate, was dominant in 72 hours over-fermented tempeh (Gunawan-Puteri et al. 2015). Tempeh fermentation also increased the fat degree of saturation, with the predominant fatty acid found in over-fermented tempeh was linoleic acid (Andriani and Nurhartadi 2014).

Microbial consortium present during the fermentation process can be very diverse and dominated by a wide variety of different types. Each type of microorganisms plays a different role which could be involved in the production of unique taste and flavor. It has been reported that Rhizopus oligosporus is the dominant fungi species in tempeh fermentation, while lactic acid and gram-negative bacteria is predominant among the bacterial community of tempeh (Mulyowidarso et al. 1990). A previous study using culture-dependent technique showed that Rhizopus sp. plays the leading role in soybean fermentation to tempeh, while the yeast and the bacteria are responsible in taste and flavor determination (Barus et al. 2008; Nurdini et al. 2015; Efriwati et al. 2017). Since most of the microorganisms were still uncultured yet, the culturedependent techniques might not be sufficient to reveal the diversity of microorganisms.

Another way to study the diversity of microorganism can be done through metagenomic analysis which is based on analysis of DNA taken directly from the environment (Jia et al. 2013). Next-Generation Sequencing (NGS) is one of the culture-independent technique for the microbial 
community metagenomic analysis. Application of NGS have resulted in the significant progress in medical microbiology for metagenomic analysis of pathogenic microorganism, the causal agents of infectious diseases (Underwood et al. 2013; Norman et al. 2014). A similar study was conducted using NGS techniques to analyze the microbial communities in the fermented food such as Korean soybean paste, doenjang (Nam et al. 2012; Kim et al. 2016).

The diversity of the microbial community in overfermented tempeh has not been reported anywhere. In this study metagenomic analysis in over-fermented tempeh using the NGS method was used to reveal the microbial diversity involved in the fermentation process.

\section{MATERIALS AND METHODS}

\section{Molecular work}

The tempeh was made from local soybean according to traditional tempeh production protocol using commercial inoculum Raprima. Soybean tempeh was further fermented for \pm 72 hours. Total DNA genome from samples was extracted using the CTAB/SDS method. DNA concentration and purity were monitored on $1 \%$ agarose gels. DNA was diluted to $1 \mathrm{ng} / \mu \mathrm{L}$ using sterile water and for reaction $1 \mathrm{ng}$ DNA was used in the PCR mixture. For PCR amplification, 16S rRNA/18SrRNA genes of distinct regions were amplified using a specific primer (16S V4: 515F-806R, 18S V4: 528F-706R) with the barcode. All PCR reactions were carried out with Phusion ${ }^{\circledR}$ HighFidelity PCR Master Mix (New England Biolabs) following the protocols from manufacturer. Final concentration of the primers used were $0.2 \mu \mathrm{M}$. The PCR products were detected in $2 \%$ agarose. Samples with a bright main strip of 400-450bp were chosen for further study. PCR products were mixed in equidensity ratios. PCR product mixtures were purified using the Qiagen Gel Extraction Kit (Qiagen, Germany). Sequencing libraries were generated using TruSeq ${ }^{\circledR}$ DNA PCR-Free Sample Preparation Kit (Illumina, USA) following the manufacturer's recommendations. The library quality was assessed on the Qubit@2.0 Fluorometer (Thermo Scientific) and Agilent Bioanalyzer 2100 system. Finally, the library was sequenced on an Illumina HiSeq 2500 platform following with $250 \mathrm{bp}$ paired-end reads generated.

\section{Data analysis}

Paired-end reads were assigned to samples based on their unique barcode and truncated by cutting of the barcode and primer sequence. Paired-end reads were merged using FLASH software (V1.2.7, http://ccb.jhu.edu/software/FLASH/) (Magoc and Salzberg 2011). Quality filtering on the raw tags was performed under specific filtering conditions to obtain the high-quality clean tags (Bokulich et al. 2013) according to the Qiime (V1.7.0, http://qiime.org/scripts/split_libraries_fastq.html) quality controlled process (Caporaso et al, 2010). The tags were compared with the reference database (Unite database, https://unite.ut.ee/) using UCHIME algorithm
(UCHIME

algorithm, http://www.drive5.com/usearch/manual/uchime_algo.html) (Edgar et al. 2011) to detect chimera sequences. Chimera sequences were then removed (Haas et al. 2011) to obtain final effective Tags.

Sequences analysis were performed using UPARSE software (UPARSE V7.0.1001, http://drive5.uparse/) (Edgar 2013). Sequences with $\geq 97 \%$ similarity were assigned to the same OTUs. A representative sequence for each OTU was screened for further annotation. For each representative sequence, the Unite Database (https://unite.ut.ee/) (Koljalg et al. 2013) was used based on Blast algorithm which was calculated by QIIME software $\begin{array}{llll}\text { (version } & 1.7 .0) \quad \text { (http://qiime.org/scripts/ }\end{array}$ assign_taxonomy.html) to annotate taxonomic information. Phylogenetic relationship construction: to study the phylogenetic relationship of different OTUs and the difference of the dominant species in different samples (groups), multiple sequence alignment were conducted using the MUSCLE software (version 3.8.31, http://www.drive5.com/muscle/) (Edgar 2004). Data Normalization: OTUs abundance information were normalized using a standard of sequence number corresponding to the sample with the least sequences. Subsequent analysis of alpha diversity and beta diversity was performed based on these output normalized data. Alpha diversity was applied in analyzing the complexity of species diversity for a sample through 6 indices, including Observed species, Chao1, Shannon, Simpson, ACE, and Good-coverage. All these indices in our samples were calculated using QIIME (version 1.7.0) and displayed using $\mathrm{R}$ software (version 2.15.3). Beta diversity on both weighted and unweighted unifrac was calculated using QIIME software (version 1.7.0).

Cluster analysis was preceded by principal component analysis (PCA), which was applied to reduce the dimension of the original variables using FactoMineR package and ggplot2 package in $\mathrm{R}$ software (version 2.15.3). Unweighted Pair-group Method with Arithmetic Means (UPGMA) Clustering was performed as a type of hierarchical clustering method to interpret the distance matrix using average linkage using QIIME software (version 1.7.0).

\section{RESULTS AND DISCUSSION}

In this research, alpha diversity was measured for the complexity analysis of a single sample. The results were summarized to access the richness and evenness of microbial community through rarefaction curves (Figure 1) and a series of indices as well (Table 1).

Table 1. Alpha diversity indices

\begin{tabular}{lcccccc}
\hline Community & Otus & Shannon Simpson & Chao1 & ACE & Coverage \\
\hline Fungi & 17 & 1.278 & 0.519 & 17.000 & 17.000 & 1.000 \\
Bacteria & 132 & 2.394 & 0.187 & 132.857 & 132.943 & 1.000 \\
\hline
\end{tabular}




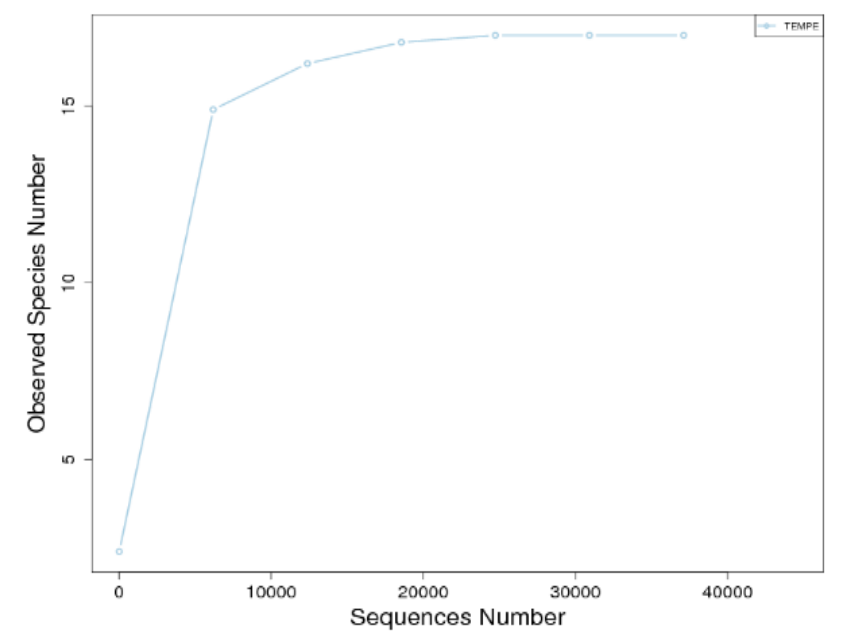

A

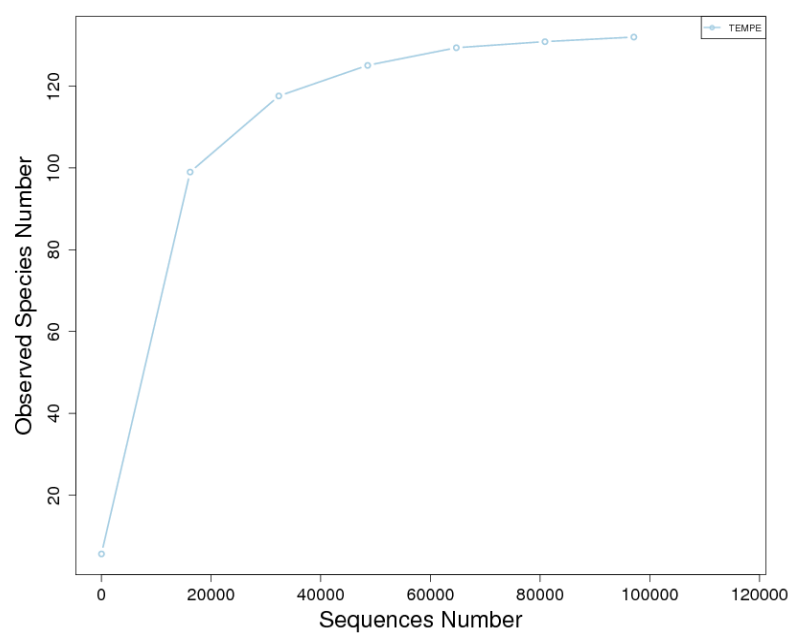

B

Figure 1. Rarefaction curves of OTUs sampling depth: A. Fungal community, B. bacterial community

The left side of the steep slope indicated that a large fraction of the species diversity remained to be discovered. If the curve becomes flattered to the right, a reasonable number of individual samples have been taken, indicating that only the rarest species remain to be sampled. All rarefaction curves in this study (Fungi and Bacterial community of over-fermented tempeh) have reached the saturation phase, indicating that most OTUs in the sample was detected and analyzed.

All metrics were calculated based on $97 \%$ identity during sequence-clustering by default. Commonly, sequences with $\geq 97 \%$ identity are to be homologous on a species level. Simpson and Shannon's indices described community diversity (Seaby and Henderson 2007), while Chao1 and Abundance-based Coverage Estimator (ACE) indices were described community richness (Chao 1984; Chao et al. 2000; Colwell 2013).

The total number of OTUs for fungi and bacterial community of over-fermented tempeh as estimated by Chao1 and ACE was similar to the observed OTUs, indicating the $100 \%$ coverage, all bacterial and fungi phylotypes presented in the sample had been covered. It was shown that the diversity of microorganism in the overfermented tempeh as estimated by Shannon and Simpson's index was higher for the bacterial community rather than that of the fungal community. Rhizopus sp. was used to make tempeh. Therefore this inoculum species was expected to be dominant in the fungal community. On the other hand, bacteria found in tempeh was seemingly from diverse endogenous microbes of soybean, so the bacterial community was more varied. Total microbial count in over-fermented tempeh was higher than in fresh tempeh, indicating that more microorganisms involved as the fermentation continued (Djunaidi et al. 2017).

Community Diversity of fungi in the sample was analyzed at each level of its taxon including kingdom, phylum, class, order, family, genus, and species. Dominant phyla were Zygomycota and Ascomycota, comprising of several families: Mucorales, Saccharomycetaceae,
Trichosporonaceae, Rhytismataceae, and Incertae sedis. Incertae sedis is a term to define taxonomic groups which are unknown of their kinship status.

Seventeen fungal OTU was found. Of 9 top OTUs, six OTUs were identified to the species level, namely Tryblidiopsis sichuanensis, Candida sp.2_1, Kluyveromyces marxianus, Trichosporon asahii, Trichosporon gracile, and Trichosporon ovoides. Two OTUs were identified in the level of the kingdom, and another one was identified in the level of order, Mucorales (Figure 5). Five species of fungi, Tryblidiopsis sichuanensis, Kluyveromyces marxianus, Trichosporon asahii, Trichosporon gracile, and Trichosporon ovoides, were never been reported to be found in Indonesian tempeh.

Phylum Zygomycota with order Mucorales showed to have the highest abundance of species in the value of $62.46 \%$. It was unsurprised because this group of fungi was used as the inoculum of tempeh. Rhizopus oligosporus was the main species being used in the preparation of tempeh, however other Rhizopus such as $R$. oryzae and $R$. formosaensis were also found from tempeh (Babu et al. 2009). This group of fungi can grow predominantly on the substrate with an abundant amount of carbon (Ferreira et al. 2012). Group of fungi from order Mucorales (Rhizopus sp., Rhizopus oligosporus, and Rhizopus oryzae) have a high hydrolytic activity such as amylolytic and proteolytic activity (Bauman and Bisping 1995; Heskamp and Barz 1998; Murashima et al. 2002; Handoyo and Morita 2006). Fungi of the order Mucorales was suggested to have a role in the degradation of complex organic compounds such as fats, proteins, and carbohydrates contained in tempeh into simple organic compounds such as fatty acids, amino acids, peptides, and monosaccharides. In addition, this group of fungi provides a substrate for bacteria in synthesizing flavors and other nutritional components. Lipolytic activity of $R$. oligosporus followed by oxidation is one of the sources of aldehydes in tempeh (Jeleń et al. 2013). Some volatile compounds are formed from amino acid 
degradation, such as sulfides, 2-methyl propanol, and 3methyl-1-butanal (Jelen et al. 2013). Ketones and 8-Calcohols, which are transformed from triglycerides and fatty acids using fungal lipoxygenase/hydroperoxylase systems, are also crucial for the aroma of tempeh (Jelen and Wasowicz 1998). Feng et al. (2007) described the formation of volatile compounds by $R$. oligosporus strains isolated from tempeh. Beside taking degradation role, Rhizopus spp. were also reported as a vitamin producer. During the solid-state fermentation of soybean, the molds incre ase the vitamin B6, riboflavin, and nicotinic acid (Bisping et al. 1993).

Efriwati et al. (2013) reported yeast occurrence in the fermentation of soybean tempeh and the diversity of yeast was depended on the method of tempeh fermentation. The predominant yeast in over-fermented tempeh, Kluyveromyces marxianus, showed to have the species abundance of $36.98 \%$. This species is grouped into proteolytic yeasts and can increase the antioxidant content (Rai et al. 2016). This proteolytic enzyme activity degrades soy protein into amino acids. This process raises the $\mathrm{pH}$. Increasing $\mathrm{pH}$ value is associated with decreasing of total acid during the further fermentation process. It was believed that this further process produced ammonia compound (Utami 2014). K. marxianus was also reported to have the ability to produce some volatile compound (Medeiros et al. 2001; Etschmann et al. 2003) that might be responsible for the over-fermented tempeh aroma.

Some species of the genus Kluyveromyces can produce a wide variety of essential enzymes, one of which is $\beta$ glucosidase (Raynal et al. 1987). During the soybean fermentation process, $\beta$-glucosidase produced could transform isoflavone compounds such as 6-O-malonyl glucoside and $\beta$-glucoside conjugate which associated with soy protein into 6-O-acetyl glucoside or $\beta$-glucoside (Barnes et al., 1998; Murphy et al., 1999; Choi et al. 2007).

During tempeh fermentation, there is the increasing of glucosidase activity resulting in a partial or total changing of glycoside level (Nout and Kiers 2005). The aglycon isoflavone compounds have a higher antioxidant activity comparing to their glycoside form because they are better absorbed in the human gastrointestinal tract (Victoria et al. 2015). Free aglycon also has an excellent antioxidant activity by blocking the LDL oxidation (Jenkins et al. 2002) and the lipid peroxidation (Wiseman 2000).

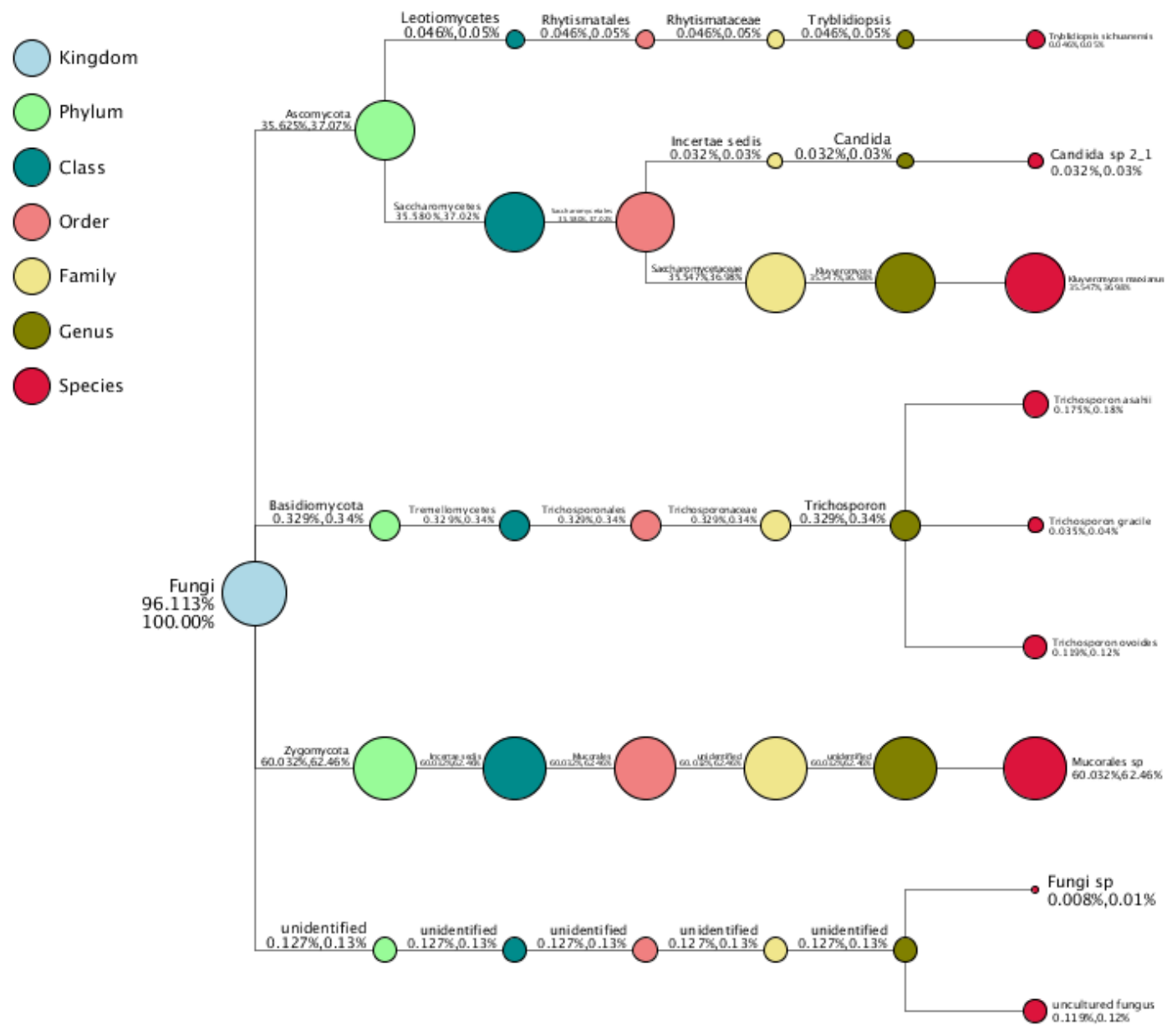

Figure 2. A diagram showing hierarchical classification of fungal species in tempeh The first number above the lines (after the taxon names) represents the relative abundance of the whole corresponding taxon, while the second number represents the relative abundance of the selected corresponding taxon 
Species Trichosporon asahii, T. ovoides, and T. gracile showed to have species abundance of $0.18 \%, 0.12 \%$, and $0.04 \%$ respectively. Trichosporon asahii and T. ovoides were reported to be found in the koji and the early period of the mash fermentation, in the soy sauce fermentation process (Wei et al. 2013). Trichosporon asahii have been reported also were found in soybean fermentation product from Korea (meju), and this species has a protease enzyme activity (Baek et al. 2014). Three species of the genus Trichosporon was suggested to have a role in the protein hydrolysis process into peptides or amino acid compounds.

In this study, two members of the class Saccharomycetes were detected: Tryblidiopsis sichuanensis and Candida sp.2_1, with species abundance of $0.05 \%$ and $0.03 \%$, respectively. These OTUs were suggested to have a role in acid fermentation in the soaking of soybean before inoculation with Rhizopus sp., but in the later stage of fermentation, these yeasts might give the over-fermented tempeh a unique aroma and flavor. The member of class Saccharomycetes contributed to the typical flavor and taste on Korean food fermentation products such as doenjang and kanjang (Haque et al. 2015). Yeast of the genus Candida, i.e., $C$. etchelsii and $C$. versatilis produced 4ethylguaiacol (4EG) and 4-ethylphenol (4EP) to give typical flavor on Japanese fermented food such as miso and shoyu (Suezawa et al. 2006; Watanabe et al. 2008).

The results of this study suggested that the bacterial community of over-fermented tempeh was diverse, in which 132 OTUs were detected. The major phyla were Firmicutes, Proteobacteria, and Bacteroidetes (Figure 3). The top 10 genera found were Chryseobacterium, Lactococcus, Lactobacillus, Streptococcus, Acetobacter, Novosphingobium, Comamonas, Escherichia, Klebsiella, and Stenotrophomonas. The dominant species, covering more than $50 \%$ of the community, were Lactobacillus agilis, Lactococcus sp., and Klebsiella sp., with the relative abundance of $27 \%, 26.3 \%$, and $13 \%$ respectively. This result is quite similar with the metagenomic study of tempeh (24 hours fermentation) which found that Firmicutes, Proteobacteria, and Bacteroidetes were the predominant phylum (Radita et al. 2017) but another phylum, Actinobacteria, was also found with minor abundance. This finding was different from the culturebased study in tempeh. Mulyowidarso et al. (1990) reported that Bacillus pumilus and B. brevis were the predominant cultured bacteria from tempeh, while some other species such as Streptococcus faecium, L. casei, K. pneumonia, and Enterobacter cloacae were also found.

Lactobacillus and Lactococcus were the most dominant group in the fermented soybean products. This group of bacteria along with yeast played essential roles during soaking of the soybean in the tempeh fermentation process which was an acid fermentation (Nurdini et al. 2015). A previous study confirming that these genera were dominant during the soybean soaking, decreased just before Rhizopus inoculation, and then re-increased post inoculation (Radita et al. 2017). The microorganisms in this acid fermentation were originated from soybean (Mulyowidarso 1989) and could survive the boiling process that this group still found in the later stages of tempeh making. The lactic acid produced decreased the $\mathrm{pH}$ and in turn, inhibited the growth of contaminating microorganisms (Ashenafi and Busse 1991). Nout and Kiers (2005) observed that the presence of $L$. plantarum on soybean inhibited both the growth of Listeria monocytogenes and staphylococcal toxin production. Lactic acid bacteria were also suggested to have hydrolytic activity and played a role in the further catabolism of amino acids and fatty acids in cheese maturation process (De Filipis et al. 2016). It seemed that this group of microorganisms might have a similar role in over-fermented tempeh, resulting in the production of specific aroma and taste.

Four OTUs of the genus Lactobacillus were detected in this study, which was closely related to L. agilis, $L$. mucosae, $L$. paralimentarius, and $L$. zeae. $L$. paralimentarius and $L$. zeae were reported as a bacterial flora of fermented food (Cai et al. 1999; Baruzzi et al. 2000). L. agilis was the dominant bacteria in the pigeon crop (Baele et al. 2001), while L. mucosae were isolated from small pig intestine (Roos et al. 2000). L. agilis also found as predominant species in fresh tempeh from Indonesia (Radita et al. 2017).

Other genera of lactic acid bacteria, Lactococcus sp., has been reported as part of bacterial flora from tempeh, ie. Lc. lactis from the soybean used as starting material and Lc. hircilactis from the soybean after the dehulling process (Radita et al. 2017). One Lactococcus in this study was only identified to the genus level. It seemed that these OTUs were genetically distinct from the isolates that have been reported previously.

Some Lactobacilli from fermented food were reported to have the ability to utilize not only oligosaccharides stachyose and raffinose but also phytic acid (Abriouel et al. 2012; Teixeira et al. 2012; Hati et al. 2013). These compounds are the component of soybean which is considered as antinutritional factors. High stachyose and raffinose cause flatulence when consumed, while phytic acid binds mineral such as phosphor. Fermentation of lupine by some lactic acid bacteria was also reported to reduce the oligosaccharide and phytic acid content (Fritsch et al. 2015).

The presence of Klebsiella pneumoniae in tempeh has been reported by some researchers (Mulyowidarso et al. 1989; Keuth and Bisping 1994). K. pneumoniae is responsible for the increase of vitamin B12 content during tempeh fermentation (Denter and Bisping 1994). $K$. pneumoniae is well-known as the pathogenic bacteria, the causative agent of pneumonia in human, but recently the isolates from Indonesia tempeh has been proven to be genetically different from the pathogenic ones (Ayu et al. 2014). In this study, 1 OTU was identified only to the level of genus Klebsiella, considering as novel species of the over-fermented tempeh. 


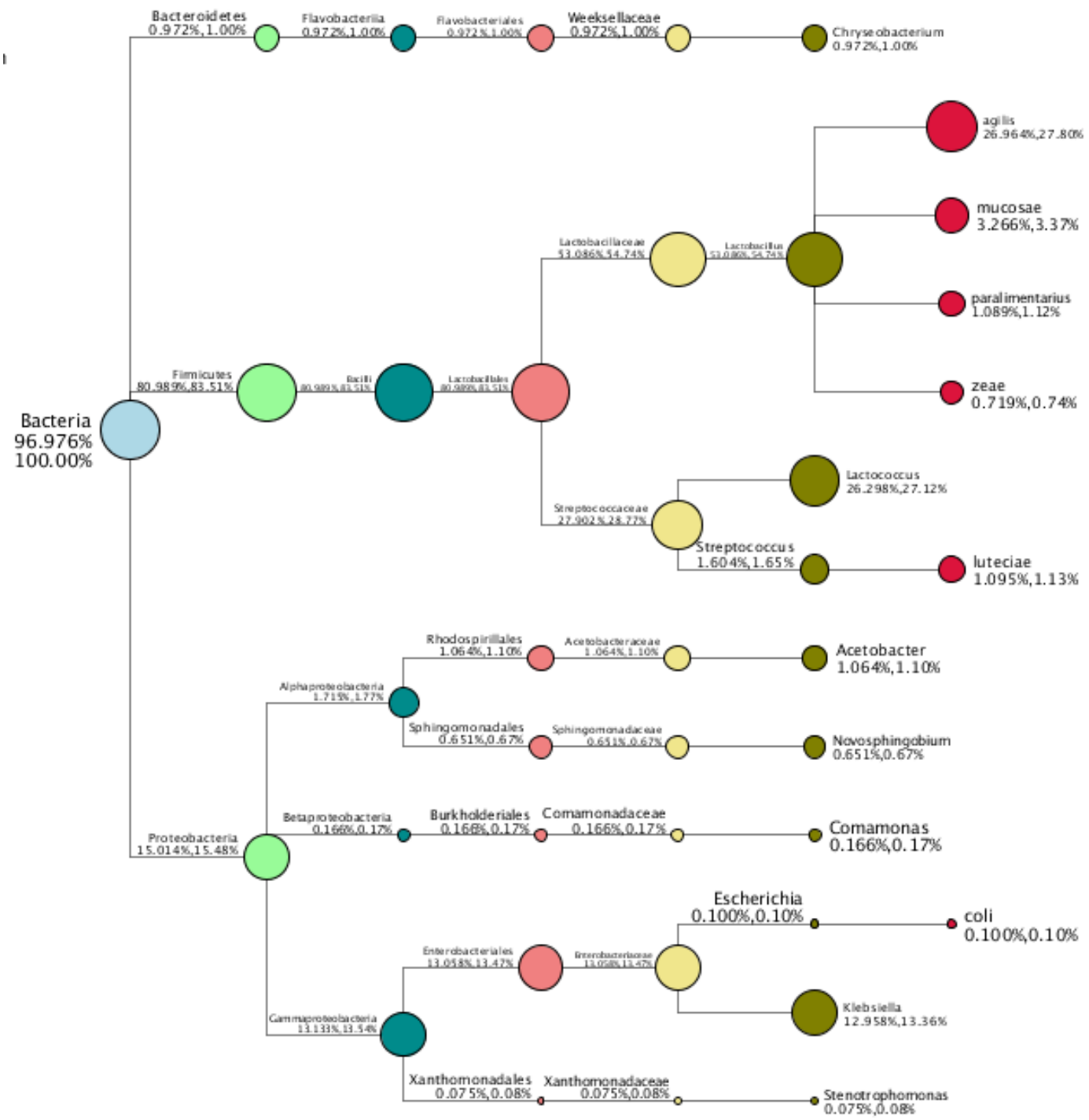

Figure 3. A diagram showing hierarchical classification of bacterial species in tempeh. The first number above the lines (after the taxon names) represents the relative abundance of the whole corresponding taxon, while the second number represents the relative abundance of the selected corresponding taxon

Other bacterial genera found in over-fermented tempeh have occurred with minor abundance, $1 \%$ and less. Acetobacter genera are the group of bacteria known as an acetic acid producer. Acetobacter is commonly found in the fermentation of vinegar. Little is known about the role of this genus in over-fermented tempeh. Barus at al. (2008) reported that Acetobacter indonesiensis was dominant in the soaking water of soybean during tempeh fermentation and was correlated with the bitter taste in tempeh produced. It is suggested that the presence of Acetobacter in overfermented tempeh contributed to the formation of specific taste.

Streptococcus luteciae is reported as part of human microbiota (Medina et al. 2017), but it has never been said to be found in fermented food. This bacteria role in
Tempeh fermentation was unclear. However, this bacteria, a lactic acid bacteria, might produce acid and have a role in acid fermentation, inhibiting contaminating microorganisms. S. lutetiensis (another name of $S$. luteciae) is reported to produce $\beta$-glucosidase (Poyart et al., 2002). $S$. lutetiensis is found as a predominant species from soybean used for tempeh making (Radita et al. 2017), showing the source of this bacteria in over-fermented tempeh.

Bacteria belonging to the genus Novosphingobium are known to be metabolically versatile, often associated with the aromatic compound degradation activity (Yan et al. 2007; Yuan et al. 2009). Novosphingobium was also reported as a member of the bacterial community in fermented food such as traditional Chinese sourdough 
(Zang and He 2013) and pickles (Jung et al. 2013). Radita et al. (2017) also found an OTU identified as Novosphingobium sp. from tempeh. This genus, along with Acetobacter and E.coli was suggested to be environmental bacteria which entered the microbial community of tempeh after the decreasing of Lactobacillus spp. caused by the dehulled-soaked soybean and washing process. These environmental bacteria came from the water used in the soaking process. The abundance of Novosphingobium sp. and $E$. coli in this study were minor, suggesting that the amount of these species decreased during the fermentation of tempeh.

Phylum Bacteroidetes had a low abundance in overfermented tempeh, approximately $1 \%$ from all taxa. This phylum was also reported to be found in fresh tempe (Radita et al. 2017) and meju, a Korean soybean fermented food (Kim et al. 2011), All with minor abundance. In this study, the only genus found from this phylum was Chryseobacterium. Genus Chryseobacterium was reported to be found in some fermented food such as Fontina cheese (Giannino et al. 2009).

Overall, the metagenomic study of the microbial community in over-fermented tempeh was successful in detecting the predominant species and could be useful to suggest their roles in tempeh fermentation. Although the tempeh microbial community depends upon the process of production which is an open fermentation process, the result was not much different from the previous study of not-over-fermented tempeh (24 hours fermentation). Thus indicated that some microbes could be a signature microbe of tempeh, regardless of the phases involved in tempeh production. For a further application, the information will help to control the process of over-fermented tempeh production as an alternative food flavoring agent which also has benefits to human health.

\section{REFERENCES}

Abriouel H, Benomar N, Cobo A, Caballero N, Fuentes MF, Pérez-Pulido R, Gálvez A. 2012. Characterization of lactic acid bacteria from naturally-fermented Manzanilla Aloreña green table olives. Food Microbiol 32 (2): 308-316.

Andriani M, Nurhartadi E. 2014. Studies on physicochemical and sensory characteristics of overripe tempeh flour as food seasoning. Acad Res Intl 5 (5): 36-45.

Ashenafi M, Busse M. 1991. Growth of Bacillus cereus in fermenting tempeh made from various beans and its inhibition by Lactobacillus plantarum. J Appl Bacteriol 70 (4): 329-333.

Ayu E, Suwanto A, Barus T. 2014. Klebsiella pneumoniae from Indonesian tempeh were genetically different from that of pathogenic isolates. Microbiol Indonesia 8 (1): 9-15.

Babu PD, Bhakyaraj R, Vidhyalakshmi R. 2009. A low-cost nutritious food 'tempeh'-A review. W J Dairy Food Sci 4 (1): 22-27.

Baek JH, So KK, Ko YH, Kim JM, Kim DH. 2014. Mycoflora and enzymatic characterization of fungal isolates in commercial meju, starter for a Korean traditional fermented soybean product. Microbiol 42 (3): 291-295.

Baele M, Devriese L, Haesebrouck F. 2001. Lactobacillus Agilis is an important component of the pigeon crop flora. J Appl Microbiol 91 (3): 488-491.

Barnes S, Coward L, Kirk M, Sfakianos J. 1998. HPLC-Mass Spectrometry analysis of isoflavones. Proc Soc Exp Biol Med 217: 254-262.
Baruzzi F, Morea M, Matarante A, Cocconcelli PS. 2000. Changes in the Lactobacillus community during Ricotta forte cheese natural fermentation. J Appl Microbiol 89 (5): 807-814.

Barus T, Suwanto A, Wahyudi AT, Wijaya H. 2008. Role of bacteria in tempe bitter taste formation and molecular biological analysis based on 16S rRNA gene. J Microbiol Indonesia 2 (1): 17-21.

Baumann U, Bisping B. 1995. Proteolysis during tempe fermentation. J Food Microbiol 12: 39-47.

Bisping B, Hering L, Baumann U, Denter J, Keuth S, Rehm HJ. 1993. Tempe fermentation: Some aspects of formation of $\gamma$-linolenic acid, proteases, and vitamins. Biotechnol Adv 11 (3): 481-493.

Bokulich NA, Subramanian S, Faith JJ, Gevers D, Gordon JI, Knight R, Mills DA, Caporaso JG. 2013. Quality filtering vastly improves diversity estimates from illumina amplicon sequencing. Nature Methods 10 (1): 57-59.

Cai Y, Okada H, Mori H, Benno Y, Nakase T. 1999. Lactobacillus paralimentarius sp. nov., isolated from sourdough. Intl J Syst Evol Microbiol 49 (4): 1451-1455.

Caporaso JG, Kuczynski J, Stombaugh J, Bittinger K, Bushman FD, Costello EK, Fierer N, Pena AG, Goodrich JK, Gordon JI, Huttley GA, Kelley ST, Knights D, Koenig JE, Ley RE, Lozupone CA, McDonald D, Muegge BD, Pirrung M, Reeder J, Sevinsky JR, Turnbaugh PJ, Walters WA, Widmann J, Yatsunenko T, Zaneveld J, rKhight R. 2010. QIIME allows analysis of high-throughput community sequencing data. Nature Methods 7 (5): 335-336.

Caplic E, Fitzgerald GF. 1999. Food fermentations: Role of microorganisms in food production and preservation. Intl $\mathrm{J}$ Food Microbiol 50: 131-149.

Chao A. 1984. Non-parametric estimation of the number of classes in a population. Scandinavian J Stat 11: 265-270.

Chao A, Hwang WH, Chen YC, Kuo CY. 2000. Estimating the number of shared species in two communities. Statistica Sinica 10: 227-246.

Choi HK, Yoon JH, Kim YS, Kwon DY. 2007. Metabolomic profiling of cheonggukjang during fermentation by ${ }^{1} \mathrm{H}$ NMR Spectrometry and Principal Components Analysis. Process Biochem 42: 263-266.

Cocolin L, Alessandria V, Dolci P, Gorra R, rRantsiou K. 2013. Cultureindependent methods to assess the diversity and dynamics of microbiota during food fermentation. Intl J Food Microbiol. 167 (1): 29-43.

Codex. 2013. Regional Standard for Tempe. Codex Alimentarius, FAO, Rome and WHO, Geneva.

Colwell RK. 2013. Statistical estimation of species richness and shared species from samples. EstimateS version 9.1.

De Filippis F, Genovese A, Ferranti P, Gilbert JA, Ercolini D. 2016. Metatranscriptomics reveals temperature-driven functional changes in microbiome impacting cheese maturation rate. Sci Rep 6: 21871.

Djunaidi S, Gunawan-Puteri MDPT, Wijaya CH, Prabawati EK. 2017. Physicochemical \& microbial characterization of overripe tempeh. INSIST 2 (1): 47.

Edgar RC. 2004. MUSCLE: Multiple Sequence Alignment with high accuracy and high throughput. Nuc Acid Res 32 (5): 1792-1797.

Edgar RC, Haas BJ, Clemente JC, Quince C, Knight R. 2011. UCHIME improves sensitivity and speed of chimera detection. Bioinformatics 27 (16): 2194-2200.

Edgar RC. 2013. UPARSE: Highly accurate OTU sequences from microbial amplicon reads. Nature Methods 10 (10): 996-998.

Efriwati, Suwanto A, Rahayu G, Nuraida L. 2013. Population dynamics of yeasts and Lactic Acid Bacteria (LAB) during tempeh production. Hayati J Biosci 20 (2): 57-64.

Etschmann MMW, Sell D, Schrader J. 2003. Screening of yeasts for the production of the aroma compound 2-phenylethanol in a molassesbased medium. Biotechnol Lett 25 (7): 531-536.

Feng XM, Volkmar P, Charlotte EJ, Marie LA, Johan S. 2007. Rhizopus oligosporus and yeast co-cultivation during barley Tempe fermentation-Nutritional impact and real-time PCR quantification of fungal growth dynamics. Food Microbiol 24: 393-402.

Ferreira JA, Lennartsson PR, Edebo L, Taherzadeh MJ. 2012. Zygomycetes-based biorefinery: present status and future prospect. Biores Tech 64 (9): 1-10.

Fritsch C, Vogel RF, Toelstede S. 2015. Fermentation performance of lactic acid bacteria in different lupin substrates-influence and degradation ability of antinutritive and secondary plant metabolites. J Appl Microbiol 119 (4): 1075-1088.

Gunawan-Puteri MDPT, Hassanein TR, Prabawati EK, Wijaya CH, Mutukumira AE. 2015. Sensory characteristics of seasoning powders 
from overripe tempeh, a solid state fermented soybean. Proc Chem 14: 263-269.

Haas BJ, Gevers D, Earl AM, Feldgarden M, Ward DV, Giannoukos G, Ciulla D, Tabbaa D, Highlander SK, Sodergren E, Methe B, DeSantis TD, Petrosino JF, Knight R, Human Microbiome Consortium, Birren BW. 2011. Chimeric 16S rRNA sequence Formation and detection in Sanger and 454 pyrosequenced PCR amplicons. Genome Res 21 (3): 494-504.

Handoyo T, Morita N. 2006. Structural and functional properties of fermented soybean (tempeh) by using Rhizopus oligosporus. Intl J Food Properties 9 (2): 347-55.

Hati S, Mandal S, Prajapati J.B. 2013. Novel starters for value-added fermented dairy products. Curr Res Nutr Food Sci J 1 (1): 83-91.

Haque MA, Seo WT, Hwang CE, Lee HY, Ahn MJ, Cho KM. 2015. Culture-independent analysis of yeast diversity in Korean traditional fermented soybean foods (doenjang and kanjang) based on 26S rRNA sequence. J Korean Soc Appl Biol Chem 58 (3): 377-385

Heskamp ML, Barz W. 1998. Expression of protease by Rhizopus species during tempeh fermentation of soybeans. Nahrung 42: 23-28.

Jia B, Xuan L, Cai K, Hu Z, Ma L, Wei C. 2013. NeSSM: A nextgeneration sequencing simulator for metagenomics. PLoS ONE 8 (10): e75448. DOI: 10.1371/journal.pone.0075448.

Jelen H, Majcher M, Ginja A, Kuligowski M. 2013. Determination of compounds responsible for tempeh aroma. Food Chem 141: 459-465.

Jelen HH, Wasowicz E. 1998.Volatile fungal metabolites and their relation to the spoilage of agricultural commodities. Food Rev Int. 4 391-426.

Jenkins DJ, Kendall CW, Jackson CJC, Connelly PW, Parker T, Faulkner D, Vidgen E, Cunnane SC, Leiter LA, Josse RG. 2002. Effects of high-and low-isoflavone soyfoods on blood lipids, oxidized LDL, homocysteine, and blood pressure in hyperlipidemic men and women. Am J Clin Nutr 76 (2): 365-372.

Jung JY, Lee SH, Jin HM, Hahn Y, Madsen EL, Jeon CO. 2013. Metatranscriptomic analysis of lactic acid bacterial gene expression during kimchi fermentation. Intl J Food Microbiol 163 (2-3): 171179.

Keuth S, Bisping B. 1994. Vitamin B12 production by Citrobacter freundii or Klebsiella pneumoniae during tempeh fermentation and proof of enterotoxin absence by PCR. Appl Environ Microbiol 60 (5): 1495-1499.

Kim MJ, Kwak HS, Jung HY, Kim SS. 2016. Microbial communities related to sensory attributes in Korean Fermented Soybean Paste (doenjang). Food Res Intl 89: 724-732

Kim YS, Kim MC, Kwon SW, Kim SJ, Park IC, Ka JO, Weon HY. 2011 Analyses of bacterial communities in meju, a Korean traditional fermented soybean bricks, by cultivation-based and pyrosequencing methods. J Microbiol 49 (3): 340-348

Koljalg U, Nilsson RH, Abarenkov K, Tedersoo L, Taylor AFS, Bahram M, Bates ST, Bruns TD, Palme JB, Callaghan TM, Douglas B, Drenkhan T, Eberhardt U, Duenas M, Grebenc T, Griffith GW, Hartmann M, Kirk PM, Kohout P, Larsson E, et al. 2013. Towards a unified paradigm for sequence-based identification of fungi. Mol Ecol 22 (21): 5271-5293.

Magoc T, Salzberg SL. 2011. FLASH: Fast Length Adjustment of Short reads to improve genome assemblies. Bioinformatics 27 (21): 2957 2963.

Medeiros ABP, Pandey A, Christen P, Fontoura PSG, de Freitas RJS, Soccol CR. 2001. Aroma compounds produced by Kluyveromyces marxianus in solid-state fermentation on a packed bed column bioreactor. World J Microbiol Biotech 17 (8): 767-771.

Medina DA, Pedreros JP, Turiel D, Quezada N, Pimentel F, Escalona A, Garrido D, 2017. Distinct patterns in the gut microbiota after surgica or medical therapy in obese patients. PeerJ 5: e3443. DOI: 10.7717/peerj.3443.

Mounier J, Monnet C, Vallaeys T, Arditi R, Santhou A, Helias A, Irlinger F. 2008. Microbial interaction within a cheese microbial community. Appl Environ Microbiol 74: 172-181.

Mulyowidarso RK, Fleet GH, Buckle KA. 1989. The microbial ecology of soybean soaking for tempe production. Intl J Food Microbiol 8 (1) $35-46$.

Mulyowidarso RK, Fleet GH, Buckle KA. 1990. Association of bacteria with the fungal fermentation of soybean tempe. J Appl Bacteriol 68 (1): 43-47.

Murashima K, Nishimura T, Nakamura Y, Koga J, Moriya T, Sumida N, Yaguchi T, Kono T. 2002. Purification and characterization of new endo-1,4-beta-d glucanases from Rhizopus oryzae. Enzyme Microbial Technol 30 (3): 319-326.

Murphy P, Song T, Buseman G, Barua K, Beecher G, Trainer D. 1999. Isoflavones in retail and institutional soy foods. J Agric Food Chem 47: 2697-2704

Nam YD, Lee SY, Lim SI. 2012. Microbial community analysis of korean soybean pastes by next-generation sequencing. Intl J Food Microbiol 155: $36-42$.

Norman JM, Handley SA, Virgin HW. 2014. Kingdom-agnostic metagenomics and the importance of complete characterization of enteric microbial communities. Gastroenterol 146: 1459-1469.

Nout MJR, Kiers JL. 2005. Tempe fermentation, innovation, and functionality: update into the third millennium. J Appl Microbiol 98 (4): 789-805.

Nurdini AL, Nuraida L, Suwanto A, Suliantari. 2015. Microbial growth dynamics during tempe fermentation in two different home industries. Intl Food Res J 22 (4): 1668-1674

Radita R, Suwanto A, Kurosawa N, Wahyudi AT, Rusmana I. 2017. Metagenome analysis of tempeh production: where did the bacterial community in tempeh come from? Malay J Microbiol 13 (4): 280-88.

Rai AK, Kumari R, Sanjukta S, Sahoo D. 2016. Production of bioactive protein hydrolysate using the yeast isolated from soft churrpi. Biores Technol 129 (7): 1-27.

Raynal AC, Gerbaud M, Francingues C, Guerineau M. 1987. Sequence and transcription of the $\beta$-glucosidase gene of Kluyveromyces fragilis cloned in Saccharomyces cerevisiae. Curr Genet 12: 175-184.

Roos S, Karner F, Axelsson L, Jonsson H. 2000. Lactobacillus mucosae sp. nov., a new species with in vitro mucus-binding activity isolated from pig intestine. Intl J Syst Evol Microbiol 50 (1): 251-258.

Seaby RMH, Henderson PA. 2007. SDR-IV Help: Measuring and Understanding Biodiversity. Pisces Conservation Ltd., Hampshire.

Suezawa Y, Kimura I, Inoue M, Gohda N, Suzuki M. 2006. Identification and typing of miso and soy sauce fermentation yeasts, Candida etchellsii and $C$. versatilis, based on sequence analyses of the D1D2 domain of the 26S Ribosomal RNA Gene, and the Region of Internal Transcribed Spacer 1, 5.8S ribosomal RNA gene and Internal Transcribed Spacer 2. Biosci Biotechnol Biochem 70: 348-354

Shurtleff W, Aoyagi A. 1979. The Book of Tempeh. Harper \& Row, London.

Poyart C, Quesne G, Trieu-Cuot P. 2002. Taxonomic dissection of the Streptococcus bovis group by analysis of manganese-dependent superoxide dismutase gene (sodA) sequences: reclassification of Streptococcus infantarius subsp. coli as Streptococcus lutetiensis sp. nov. and of Streptococcus bovis biotype 11.2 as Streptococcus pasteurianus sp. nov. Intl J Syst Evol Microbiol 52 (4): 1247-1255.

Teixeira JS, McNeill V, Gänzle MG. 2012. Levansucrase and sucrose phosphorylase contribute to raffinose, stachyose, and verbascose metabolism by lactobacilli. Food Microbiol 31 (2): 278-284.

Underwood AP, Dallman T, Thomson NR, Williams W, Harker K, Perry N, Adak B, Willshaw G, Cheasty T, Green J, Dougan G, Parkhill J, Wain J. 2013. Public health value of next-generation DNA sequencing of enterohemorrhagic Escherichia coli isolates from an outbreak. J Clin Microbiol 51: 232-237.

Utami R, Wijaya CH, Lioe HN. 2016. Taste of water-soluble extracts obtained from over-fermented tempe. Intl J Food Properties 19 (9): 2063-2073.

Victoria BG, Rodica S., Georgeta NC, 2015. Improvement of the antioxidant activity of soybean (Glycine $\max$ ) by biotechnological processing. Rom Biotechnol Lett 20 (2): 10212-10220.

Wei Q, Wang H, Chen Z, Lu Z, Xie Y, Lu F. 2013. Profiling of dynamic changes in the microbial community during the soy sauce fermentation process. Appl Microbiol Biotech 97: 9111-9119.

Watanabe Y, Nagayama K, Tamai Y. 2008. Expression of Glycerol 3Phosphate Dehydrogenase Gene (CvGPDI) in salt-tolerant yeast Candida versatilis is stimulated by high concentrations of $\mathrm{NaCl}$. Yeast 25: 107-116.

Wei CL, Chao SH, Tsai WB, Lee PS, Tsau NH, Chen JS, Lai WL, Tu JCY, Tsai YC. 2013. Analysis of bacterial diversity during the fermentation of inyu, a high-temperature fermented soy sauce, using nested PCR-denaturing gradient gel electrophoresis and the plate count method. Food Microbiol 33 (2): 252-261.

Wiseman H, O'Reilly JD, Adlercreutz H, et al. 2000. Isoflavone phytoestrogens consumed in soy decrease F2-isoprostane concentrations and increase resistance of low-density lipoprotein to oxidation in humans. Am J Clin Nutr 72: 395-400 
Yan QX, Hong Q, Han P, Dong XJ, Shen YJ, Li SP. 2007. Isolation and characterization of a carbofuran-degrading strain Novosphingobium sp. FND-3. FEMS Microbiol Lett 271 (2): 207-213.

Yuan J, Lai Q, Zheng T, Shao Z. 2009. Novosphingobium indicum sp. nov., a polycyclic aromatic hydrocarbon-degrading bacterium isolated from a deep-sea environment. Intl J Syst Evol Microbiol 59 (8): 2084 2088.

Zhang G, He G. 2013. Predominant bacteria diversity in Chinese traditional sourdough. J Food Sci 78 (8): M1218-M1223. 